\title{
Normal form in Hecke-Kiselman monoids associated with simple oriented graphs*
}

\author{
R. Aragona and A. D'Andrea \\ Communicated by V. Mazorchuk
}

\begin{abstract}
A Bstract. We generalize Kudryavtseva and Mazorchuk's concept of a canonical form of elements [9] in Kiselman's semigroups to the setting of a Hecke-Kiselman monoid $\mathbf{H K}_{\Gamma}$ associated with a simple oriented graph $\Gamma$. We use confluence properties from [7] to associate with each element in $\mathbf{H K}_{\Gamma}$ a normal form; normal forms are not unique, and we show that they can be obtained from each other by a sequence of elementary commutations. We finally describe a general procedure to recover a (unique) lexicographically minimal normal form.
\end{abstract}

\section{Introduction}

Let $\Gamma$ be a simple mixed graph, i.e., each pair of distinct vertices in $\Gamma$ has at most one connection, which can be either oriented or unoriented; thus, there are no oriented cycles of length two and no vertex in $\Gamma$ has a self loop.

One may use [5] the combinatorial content of $\Gamma$ to give a presentation of a Hecke-Kiselman semigroup $\mathbf{H K}_{\Gamma}$. If $V$ is the set of vertices of $\Gamma, \mathbf{H K}_{\Gamma}$ is generated by idempotent elements $a_{i}, i \in V$, which satisfy the relations:

- $a_{i} a_{j}=a_{j} a_{i}$ if there is no connection between the vertices $i, j \in V$;

${ }^{*}$ Both authors are members of INdAM-GNSAGA. The second named author was partially supported by Ateneo fundings from Sapienza University in Rome.

2010 MSC: 20M05, 20M10.

Key words and phrases: simple oriented graph, Hecke-Kiselman monoid, normal form. 
- $a_{i} a_{j} a_{i}=a_{j} a_{i} a_{j}$ if there is an unoriented connection between $i$ and $j$;

- $a_{i} a_{j} a_{i}=a_{j} a_{i} a_{j}=a_{i} a_{j}$ if there is an arrow connecting $i$ to $j$.

If $\Gamma$ is an unoriented simple graph, i.e., if relations in the above presentation are all of the first two kinds, then we obtain the Coxeter monoid associated to the simply laced Dynkin diagram $\Gamma$. This is also known in the literature either as Richardson-Springer [15] or 0-Hecke monoid [6], as its monoid algebra [13] may be obtained as the $q=0$ specialization of a Iwahori-Hecke algebra.

The third type of relation has been first observed by Kiselman [8]. When $\Gamma=\Gamma_{n}$ is the graph on the vertex set $\{1,2, \ldots, n\}$ with a single oriented connection between $i$ and $j$ each time that $i<j$, one obtains the so-called Kiselman semigroups, so that Kiselman's original example corresponds to $\Gamma_{3}$. These semigroups also occur in the study [3] of some graph-dynamical systems related to SDS [2].

Understanding which mixed graphs $\Gamma$ yield finite Hecke-Kiselman monoids is a difficult problem and the only nontrivial results so far seem to be [1] and $[10,11]$. In the same vein, a characterization of reduced expressions of elements as words in the idempotent generators are only known in the Kiselman case $\Gamma=\Gamma_{n}$ [9] or when $\Gamma$ is an unoriented graph and one may reduce to standard Coxeter combinatorics. The present paper deals with the easier case where only oriented connections occur. We should stress that our result is implicit in [5], where the focus is on equioriented Dynkin graphs of type $A_{n}$, and in [14], where a Gröbner basis approach is used, though only the case where $\Gamma$ is an oriented cycle of length $n$ is mentioned.

We employ Huet's reformulation [7] of Newman's results [12] to extend the strategy outlined by Kudryavtseva and Mazorchuk [9] for Kiselman's semigroups, to all (possibly infinite) Hecke-Kiselman monoids corresponding to simple oriented graphs. The concrete statement is that normal forms of each element in $\mathbf{H K}_{\Gamma}$ all arise via a decreasing sequence of cancellations, that all decreasing sequences of cancellations may be continued to a normal form, that such normal forms all have the same length, and may be obtained from each other by a sequence of elementary commutations between pairs of disconnected idempotent generators. We end the paper with some final comments on how to select a lexicographically minimal normal form. 


\section{Normal Form in $\mathbf{H K}_{\Gamma}$}

In what follows, $\Gamma=(V, E)$ will be a simple oriented graph, i.e., a directed graph that does not have oriented cycles of length 1 or 2 , so that there are no self-loops and there is at most one connection between two given vertices. Here $V$ denotes the (possibly infinite) vertex set and $E \subseteq V \times V$ is the arrow set, where $(a, b) \in E$ if and only if there is an arrow connecting $a$ to $b$; indeed, we will use the shorthand notation $a \longrightarrow b$ as equivalent to $(a, b) \in E$. Notice, however, that we will spare the symbol $\longrightarrow$ for a different context, in order to adhere to notations from [7].

Any given choice of $\Gamma$ yields a Hecke-Kiselman monoid $\mathbf{H K}_{\Gamma}$ defined by the presentation

$$
\begin{aligned}
\mathbf{H K}_{\Gamma}=\langle a \in V| & a^{2}=a, \text { for every } a \in V ; \\
& a b a=b a b=a b, \text { if } a \longrightarrow b ; \\
& a b=b a, \text { if } a \nrightarrow b \text { and } b \nrightarrow a\rangle .
\end{aligned}
$$

If we denote by $F(V)$ the free monoid on the alphabet $V$, then we have a canonical projection

$$
\pi: F(V) \rightarrow \mathbf{H K}_{\Gamma} .
$$

Every $a \in V \subseteq F(V)$ will be called a letter; if $w \in F(V)$ is obtained by multiplying letters among which $a$ occurs, we will say that (the word) $w$ contains (the letter) $a$, or that $a$ occurs in $w$. The same terminology will be used when $w \in \mathbf{H K}_{\Gamma}$; this is well defined as the letter content in both sides of each relation presenting $\mathbf{H K}_{\Gamma}$ is the same, so that all words in $F(V)$ projecting via $\pi$ to the same element of $\mathbf{H K}_{\Gamma}$ have the same letter content. Note that each letter in $\mathbf{H K}_{\Gamma}$ is idempotent.

Remark 1. $\mathbf{H K}_{\Gamma}$ is finite if and only if $\Gamma$ is finite and acyclic [1].

First, for the sake of completeness, we give a proof of the following well known result (see for instance [4,11]).

Lemma 1. If $a \in \mathbf{H K}_{\Gamma}$ is a letter and $w \in \mathbf{H K}_{\Gamma}$ is obtained by multiplying letters that do not admit arrows to (respectively from) $a$, then awa $=$ aw (resp. awa $=w a)$.

Proof. If $a$ and $b$ are letters in $\mathbf{H K}_{\Gamma}$ such that $b \nrightarrow a$, then either $a \longrightarrow b$, whence $a b a=a b$, or $a b=b a$, whence $a b a=a(b a)=a(a b)=a^{2} b=a b$. Also, if $u$ and $v$ are words in $\mathbf{H K}_{\Gamma}$ satisfying respectively $a u a=a u$ 
and $a v a=a v$, then $a u v a=(a u) v a=(a u a) v a=a u(a v a)=a u(a v)=$ $(a u a) v=a u v$. Now the statement follows by an easy induction.

The case where $w$ is obtained by multiplying letters that do not admit arrows from $a$ is done similarly.

Let $w_{1}, w_{2}, u \in F(V)$. It is useful to introduce the following elementary cancellations on words in $F(V)$.

- Right cancellation: $w_{1} a u a w_{2} \stackrel{r}{\longrightarrow} w_{1} a u w_{2}$, if $a$ is a letter and no letter in $u$ has an arrow to $a$;

- Left cancellation: $w_{1} a u a w_{2} \stackrel{l}{\longrightarrow} w_{1} u a w_{2}$, if $a$ is a letter and no letter in $u$ has an arrow from $a$.

Without loss of generality, we may assume above that $u$ does not contain the letter $a$ and only focus on elementary cancellations between consecutive occurrences of the same letter. Notice that if $v$ is obtained from $w$ by a sequence of elementary cancellations, then $v, w \in F(V)$ map to the same element in $\mathbf{H K}_{\Gamma}$.

Remark 2. Idempotence of letters and each relation $a b a=b a b=a b$ in the presentation of $\mathbf{H K}_{\Gamma}$ are special instances of elementary cancellations. Thus, elementary cancellations along with commutations of disconnected letters provide an equivalent presentation of $\mathbf{H K}_{\Gamma}$.

Definition 1. Let $v, w \in F(V)$. We shall write by $w \stackrel{*}{\longrightarrow} v$ if $v$ is obtained from $w$ by a (possibly empty) sequence of (either right or left) elementary cancellations. In other words, $\stackrel{*}{\longrightarrow}$ is the reflexive-transitive closure of the relation $\longrightarrow=\stackrel{r}{\longrightarrow} \cup \stackrel{l}{\longrightarrow}$ on $F(V)$.

A simplifying sequence (with respect to $\Gamma$ ) from $w$ to $v$ is a sequence of elementary cancellations which transform $w$ into $v$. This is analogous to [9, Remark 7]. We take the following definition from [7] .

Definition 2. A word $w \in F(V)$ is a normal form for $\pi(w) \in \mathbf{H K}_{\Gamma}$ if no elementary cancellation may be performed on $w$.

We shall denote by $\mathcal{N}$ the set of all normal forms in $F(V)$. Notice that $\mathcal{N}$ depends on $\Gamma$, which we consider to be fixed once and for all.

Remark 3. By Lemma 1, $w \in F(V)$ is a normal form for $\pi(w) \in \mathbf{H K}_{\Gamma}$ if and only if each subword of $w$ of the form aua, where $a \in V$ and $u \in F(V)$ does not contain $a$, contains at least one letter with an arrow to $a$ and at least one letter with an arrow from $a$. In [5] these words are called special when $\Gamma=\Gamma_{n}$ and strongly special when $\Gamma$ is an equioriented Dynkin diagram of type $A_{n}$. 
Note that, by definition, if $\gamma \in \mathbf{H K}_{\Gamma}$, then any word $w \in \pi^{-1}(\gamma)$ of minimal length is a normal form of $\gamma$ so, in particular, each $\gamma \in \mathbf{H K}_{\Gamma}$ admits at least one normal form. However, in principle, a normal form of $\gamma \in \mathbf{H K}_{\Gamma}$ may fail to be of minimal length. We will show that this is not the case by proving that all normal forms of $\gamma$ share the same length and, more precisely, that they can be obtained from each other by a sequence of commutations between disconnected letters.

Recall that [9, Theorem 6] exploits Newman's Diamond Lemma [12] in the case of the complete oriented acyclic $\Gamma_{n}$, so as to show that:

(1) each $\gamma \in \mathbf{H K}_{\Gamma_{n}}$ has a unique normal form;

(2) every word $w \in \pi^{-1}(\gamma)$ is connected to the unique normal form for $\gamma$ by a simplifying sequence;

(3) each simplifying sequence starting from $w$ may be completed to a sequence as in (2).

Claim (1) may certainly fail in our generalized setting. Indeed if $a, b \in \mathbf{H K}_{\Gamma}$ are commuting letters then $a b$ and $b a$ are distinct normal forms for the same element in $\mathbf{H K}_{\Gamma}$. We want to show that this is basically the only obstruction to uniqueness.

Definition 3. We denote by $\sim$ the equivalence relation on $F(V)$ generated by elementary commutations

$$
w_{1} a b w_{2} \sim w_{1} b a w_{2}
$$

where $w_{1}, w_{2} \in F(V)$ and $a, b \in V$ are disconnected letters, i.e., they satisfy $a \nrightarrow b$ and $b \nrightarrow a$.

Our strategy is to use confluence properties of the relation $\longrightarrow$ modulo the equivalence $\sim$ on $F(V)$. In order to do so, we set ourselves within the framework described by Huet in [7, Section 2.3] to make sure that the possibility to apply any given elementary cancellation on $w \in F(V)$ only depends on its -equivalence class; furthermore that such elementary cancellations yield $\sim$-equivalent words.

Lemma 2. Let $v, w \in F(V)$, and assume that $w \stackrel{*}{\longrightarrow} v$. If $\widetilde{w} \sim w$, then there exists $\widetilde{v} \sim v$ such that $\widetilde{w} \stackrel{*}{\longrightarrow} \widetilde{v}$.

Proof. It suffices to only treat the case where $\widetilde{w}$ is obtained from $w$ by a single elementary commutation and $v$ is obtained from $w$ by a single elementary cancellation.

Set $w=w_{1} a u a w_{2}$ and $v=w_{1} a u w_{2}$, where no letter in $u$ has an arrow to $a$. Let $\widetilde{w}$ be obtained from $w$ by means of an elementary commutation; 
if this occurs outside aua or within $u$, then the claim is clear. The only possibly nontrivial case is when the elementary commutation involves either the leading or the ending letter in subword aua. By assumption, $u$ does not contain the letter $a$, and without loss of generality we may also assume that the elementary commutation involves a letter of $u$. We have two cases:

(i) $u=b u^{\prime}$ and the elementary commutation involves $a$ and $b$. Then $w=w_{1} a b u^{\prime} a w_{2}$ and $\widetilde{w}=w_{1} b a u^{\prime} a w_{2}$. Notice that as $u^{\prime}$ is a subword of $u$, no letter from $u^{\prime}$ has an arrow to $a$. Thus we may perform a right cancellation on the subword $a u^{\prime} a$ giving $\widetilde{v}=w_{1} b a u^{\prime} w_{2}$. However, $\widetilde{v}$ is obtained from $v=w_{1} a b u^{\prime} w_{2}$ by elementary commutation of $a$ with $b$.

(ii) $u=u^{\prime} b$ and the elementary commutation involves $b$ and $a$. Then $w=w_{1} a u^{\prime} b a w_{2}$ and $\widetilde{w}=w_{1} a u^{\prime} a b w_{2}$. Once more we may perform a right cancellation on the subword $a u^{\prime} a$ giving $\widetilde{v}=w_{1} a u^{\prime} b w_{2}$, which coincides with $v$.

The proof for left cancellations is completely analogous.

Remark 4. - By Lemma 2, each element in the $\sim$-equivalence class of a normal form is also a normal form.

- If $u$ only contains letters that are not connected to the letter $a$, then both a right and a left cancellation may be performed on $w_{1} a u a w_{2}$. However, the resulting words $w_{1} a u w_{2}$ or $w_{1} u a w_{2}$ lie in the same $\sim$-equivalence class.

\section{Normal Forms and confluence}

Let us consider the framework of [7, Section 2.3], where, in our setting, - $\mathscr{E}$ is the free monoid $F(V)$ on $V$,

- $\longrightarrow$ is the binary relation $\stackrel{r}{\longrightarrow} \cup \stackrel{l}{\longrightarrow}$ on $F(V)$,

- $\stackrel{*}{\longrightarrow}$ is the reflexive-transitive closure of the relation $\longrightarrow$ on $F(V)$,

- $\sim$ is the equivalence relation on $F(V)$ generated by elementary commutations of disconnected letters, and

- $x \equiv y$ if and only if $\pi(x)=\pi(y)$, where $\pi: F(V) \rightarrow \mathbf{H K}_{\Gamma}$ is the canonical projection. Indeed, by Remark 2 , the equivalence relation $\equiv$ generated by $\longrightarrow \cup \sim$, as from [7, Lemma 2.6], coincides with the quotient relation induced by the presentation of $\mathbf{H K}_{\Gamma}$.

We will need to verify if the relation $\longrightarrow$ on $F(V)$ satisfies the following properties, given in [7]. 
Confluence modulo $\sim$. For all choices of $x \sim y, x^{\prime}, y^{\prime} \in F(V)$ such that $x \stackrel{*}{\longrightarrow} x^{\prime}, y \stackrel{*}{\longrightarrow} y^{\prime}$, one may find $\bar{x}, \bar{y}$ such that

$$
x^{\prime} \stackrel{*}{\longrightarrow} \bar{x}, \quad y^{\prime} \stackrel{*}{\longrightarrow} \bar{y}, \quad \bar{x} \sim \bar{y} .
$$

Locally confluence modulo $\sim$. The following conditions are satisfied

$\alpha$ : for all $x, y, z \in F(V)$ such that $y$ and $z$ are obtained from $x$ by any elementary cancellation, then there exist $u, v \in F(V)$ such that $y \stackrel{*}{\longrightarrow} u, z \stackrel{*}{\longrightarrow} v$ and $u \sim v$;

$\beta$ : for all $x, y, z \in F(V)$ such that $x \sim y$ and $z$ is obtained from $x$ by any elementary cancellation, then there exist $u, v \in F(V)$ such that $y \stackrel{*}{\longrightarrow} u, z \stackrel{*}{\longrightarrow} v$ and $u \sim v$.

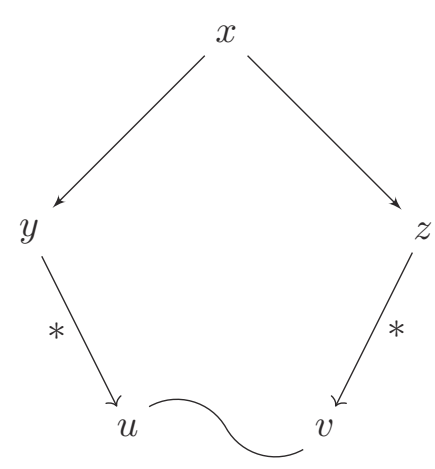

Condition $\alpha$

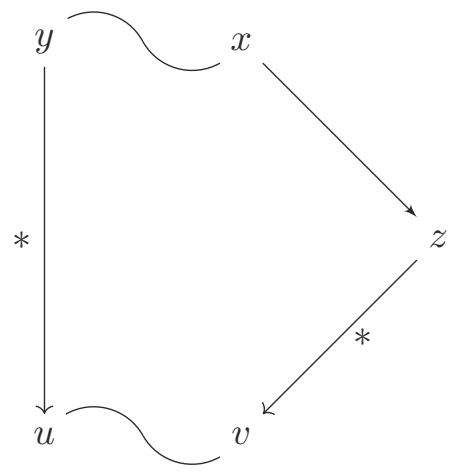

Condition $\beta$

Since each elementary cancellation decreases word length, the relation $\longrightarrow$ is noetherian [7, Section 2.1], i.e., there is no infinite sequence of elementary cancellations. In the noetherian case, [7, Lemma 2.7] shows that confluence modulo $\sim$ and local confluence modulo $\sim$ are equivalent.

The following theorem proves that each normal form of a word $w \in$ $\pi^{-1}(\gamma)$ belongs to the same $\sim$-equivalence class and it follows that all the normal forms of $w$ have the same length. In particular we obtain that the number of simplifying steps to achieve a normal form of $\pi(w)$ starting from $w$ is independent of the chosen simplifying sequence.

Theorem 1. 1) Let $x, y \in F(V)$. If $\pi(x)=\pi(y)$ and $u, v \in \mathcal{N}$ satisfy $x \stackrel{*}{\longrightarrow} u$ and $y \stackrel{*}{\longrightarrow} v$. Then $u \sim v$.

2) Every simplifying sequence

$$
x \longrightarrow x_{1} \longrightarrow x_{2} \longrightarrow \cdots \longrightarrow x_{n}
$$

may be extended to a simplifying sequence ending on a normal form of $\pi(x)$. 
3) All simplifying sequences starting from $x \in F(V)$ and ending on some normal form of $\pi(x)$ have the same length.

Proof. The second claim is a rephrasing of the concept of normal form, whereas the third claim follows immediately from the first one, once we notice that words in the same $\sim$-equivalence class have the same length and each elementary cancellation decreases word length by exactly one.

As for the first claim, this is just [7, Lemma 2.6], which is equivalent to $\longrightarrow$ being confluent modulo $\sim$. As we are in a noetherian setting, it is enough to prove that local confluence holds, i.e., that conditions $\alpha$ and $\beta$ are satisfied.

Condition $\alpha$ follows from [9, Lemma 8], where Kudryavtseva and Mazorchuk, more generally, prove that for all $x, y, z \in F(V)$ such that $y$ and $z$ are obtained from $x$ by any elementary cancellation, then there exists $u \in F(V)$ such that $y \stackrel{*}{\longrightarrow} u$ and $z \stackrel{*}{\longrightarrow} u$.

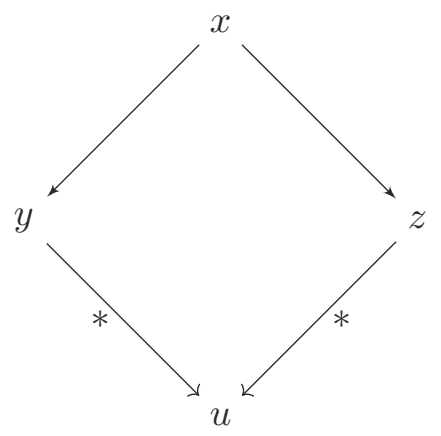

Condition $\beta$ follows from the fact that, by Lemma 2 , for all $x, y, z \in$ $F(V)$ such that $x \sim y$ and $z$ is obtained from $x$ by any elementary cancellation, there exists $u \in F(V)$ such that $z \sim u$ and $u$ is obtained from $y$ by an elementary cancellation.

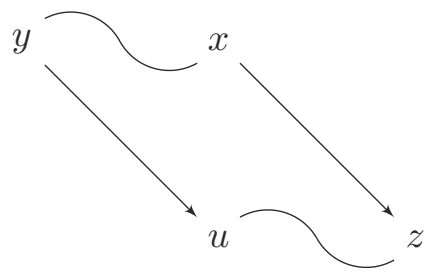

Corollary $\mathbf{1}$ ([9, Theorem 6]). Normal forms in $\mathbf{H K}_{\Gamma_{n}}$ are unique.

Proof. If $u$ and $v$ are normal forms of the same element in $\mathbf{H K}_{\Gamma}$, then $u \sim v$. However, $\sim$ is the trivial equivalence relation when $\Gamma=\Gamma_{n}$. 
The next corollary is a special case of [5, Proposition 15]. Here we only give an alternative proof.

Corollary 2. Let $\Gamma$ be a (possibly infinite) simple oriented graph, $\Gamma^{\prime} \subset \Gamma$ a full subgraph. Then the induced monoid homomorphism $\mathbf{H K}_{\Gamma^{\prime}} \rightarrow \mathbf{H K}_{\Gamma}$ is injective.

Proof. Normal forms of words whose letter content lies in $\Gamma^{\prime}$ are the same in both monoids.

Remark 5. We stress the fact that Theorem 1 uses neither finiteness of the graph $\Gamma$ nor that of the monoid $\mathbf{H K}_{\Gamma}$. For instance, when $\Gamma$ has an oriented cycle, one may prove that $\mathbf{H K}_{\Gamma}$ is infinite [1] by noticing that each power of the ordered product of all letters in the cycle is a normal form, hence they describe infinitely many distinct elements, as they have distinct length.

\section{Choosing a preferred normal form}

In actual contexts one would like to locate a favorite normal form to work with. One way to do this is by choosing a total ordering $<$ on the set $V$ of the vertices of $\Gamma$ and employ the induced lexicographic ordering on $F(V)$ so as to choose the minimal normal form.

If $[w]$ is the $\sim$-equivalence class of a normal form for some $\gamma \in \mathbf{H K}_{\Gamma}$, we will henceforth denote by $w^{\text {min }}$ its lexicographically minimal element. In principle, one may not be able to obtain $w^{\min }$ from $w$ by a sequence of lexicographically decreasing elementary commutations.

Example 1. Consider the total ordering $a<b<c$ on the graph

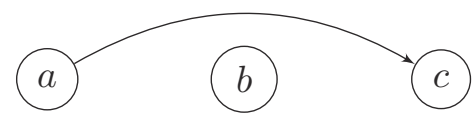

If $w=c a b$, then $[w]=\{b c a, c a b, c b a\}$ so that $w^{\min }=b c a$. Elementary commutations all involve $b$, so that the only way to commute $w$ into $w^{\text {min }}$ is $c a b \sim c b a \sim b c a$; however $c a b$ is lexicographically lower than $c b a$.

We thus need to find a general strategy to recover $w^{\min }$ from $w$.

Definition 4. Let $a_{i} \in V, i=1, \ldots, n$, so that $w=a_{1} a_{2} \ldots a_{n} \in F(V)$. Then $a_{j}$ is an initial letter of $w$, if $a_{j}$ commutes with $a_{i}$ for each $i<j$. 
Denote now by $\iota(w)$ the least initial letter of $w$. We are going to describe a procedure to select a lexicographically minimal normal form for any element in $\mathbf{H K}_{\Gamma}$.

Proposition 1. Let $w \in F(V)$ be a normal form. If $w^{\min }=a_{1} a_{2} \ldots a_{n}$, $a_{i} \in V$, then for all $k \geqslant 0, a_{k+1}$ is the least initial letter of the word $w_{k}$ obtained from $w$ by removing the leftmost occurrences of the letters $a_{1}, a_{2}, \ldots, a_{k}$. Equivalently, $w^{\min }=\iota(w) w_{1}{ }^{\min }$.

Proof. First of all, by the very definition, initial letters of $w$ all commute with which other. Also, if $w^{\prime}$ is obtained from $w$ by an elementary commutation then the sets of initial letters of $w$ and $w^{\prime}$ coincide; therefore, the set of initial letters of a word only depends on its -equivalence class. Every initial letter of $w$ may be commuted to the leftmost position; vice versa the leading letter of each word in the $\sim$-equivalence class of $w$ is an initial letter for $w$. Thus $\iota(w)$ is the leftmost letter of $w^{\text {min }}$. Now, $w^{\text {min }}=\iota(w) w_{1}{ }^{\text {min }}$ can be easily proved by induction on the length $n$ of $w$.

Corollary 3. Define inductively a word $w \in F(V)$ to be tidy as follows:

- the empty word is tidy;

- $w$ is tidy if $w=\iota(w) w_{1}$ and $w_{1}$ is tidy.

Then there exists a bijection between $\mathbf{H K}_{\Gamma}$ and the set of tidy normal forms, which associates with every element $\gamma \in \mathbf{H K}_{\Gamma}$ its unique lexicographically minimal normal form.

The above claims shows that $w^{\text {min }}$ can be recursively computed from $w$. Notice, however, that the actual computation of $w^{\text {min }}$ will strongly depend on the topology of $\Gamma$. For instance, when $\Gamma=\Gamma_{n}$, no elementary commutations will be needed at all, so that $w$ and $w^{\min }$ will always coincide. The opposite extreme is when $\Gamma$ is a totally disconneted graph; in this case a word is normal form if and only if each of its letters only occurs once. One then obtains $w^{\text {min }}$ from $w$ by sorting $w$ with respect to the chosen total order. Intermediate cases will require some "partial sorting" of the letter content of $w$. How to do this efficiently seems to be an interesting problem, which will be addressed in a future paper.

\section{References}

[1] R. Aragona and A. D'Andrea, Hecke-Kiselman monoids of small cardinality. Semigroup Forum 86, 2013, pp. 32-40.

[2] C. L. Barrett, H. S. Mortveit, and C. M. Reidys, Elements of a theory of simulation. II. Sequential dynamical systems, Appl. Math. Comput. 107, 2000, pp.121-136. 
[3] E. Collina and A. D'Andrea, A graph-dynamical interpretation of Kiselman's semigroups, J. Alg. Comb. 41, 2015, pp. 1115-1132.

[4] L. Forsberg, Effective representations of Hecke-Kiselman monoids of type A, preprint arXiv: $1205.0676 \mathrm{v} 4$.

[5] O. Ganyushkin and V. Mazorchuk, On Kiselman quotients of 0-Hecke monoids, Int. Electron. J. Algebra 10, 2011, pp. 174-191.

[6] F. Hivert, A. Schilling, N.M. Thiéry, The biHecke monoid of a finite Coxeter group, 22nd International Conference on Formal Power Series and Algebraic Combinatorics (FPSAC 2010), pp. 307-318, Discrete Math. Theor. Comput. Sci. Proc., 2010.

[7] G. P. Huet, Confluent Reductions: Abstract Properties and Applications to Term Rewriting Systems, Journal of the ACM 27, 1980, pp. 797-821.

[8] C. O. Kiselman, A semigroup of operators in convexity theory, Trans. AMS 354, 2002, pp. 2035-2053.

[9] G. Kudryavtseva and V. Mazorchuk, On Kiselman's semigroup, Yokohama Math. J. 55, 2009, pp. 21-46.

[10] A. Męcel and J. Okniński, Growth alternative for Hecke-Kiselman monoids, Publ. Mat. 63, 2019, pp. 219-240.

[11] A. Męcel and J. Okniński, Gröbner basis and the automaton property of HeckeKiselman algebras, Semigroup Forum 99, 2019, pp. 447-464.

[12] M. H. A. Newman, On theories with a combinatorial definition of "equivalence", Ann. of Math. 43, 1942, pp. 223-243.

[13] P. N. Norton, 0-Hecke algebras, J. Austral. Math. Soc. (Series A) 27, 1979, pp. 337-357

[14] J. Okniński, M. Wiertel, Combinatorics and structure of Hecke-Kiselman algebras, preprint arXiv:1904.12202.

[15] R. Richardson and T. Springer, The Bruhat order on symmetric varieties, Geom. Dedic. 35, 1990, pp. 389-436.

\section{CONTACT INFORMATION}

Riccardo Aragona DISIM, University of L'Aquila, Via Vetoio, Coppito - 67100 L'Aquila, Italy E-Mail(s): riccardo.aragona@univaq.it
Alessandro
Department of Mathematics "Sapienza"
D'Andrea
University of Rome, P.le Aldo Moro, 5 - 00185
Rome, Italy
E-Mail(s): dandrea@mat.uniroma1.it

Received by the editors: 26.03.2020

and in final form 07.12.2020. 